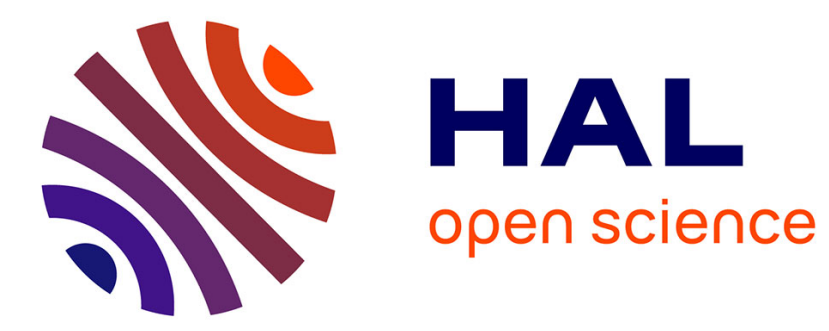

\title{
Intérêt et limites de la production de viandes de porc mâle entier
}

Michel Bonneau

\section{To cite this version:}

Michel Bonneau. Intérêt et limites de la production de viandes de porc mâle entier. Productions Animales, 1988, 1 (2), pp.133-140. hal-00895825

\section{HAL Id: hal-00895825 \\ https://hal.science/hal-00895825}

Submitted on 1 Jan 1988

HAL is a multi-disciplinary open access archive for the deposit and dissemination of scientific research documents, whether they are published or not. The documents may come from teaching and research institutions in France or abroad, or from public or private research centers.
L'archive ouverte pluridisciplinaire HAL, est destinée au dépôt et à la diffusion de documents scientifiques de niveau recherche, publiés ou non, émanant des établissements d'enseignement et de recherche français ou étrangers, des laboratoires publics ou privés. 
INRA Prod. Anim.,

1988, 1 (2), 133-140

\section{BONNEAU}

INRA Saint-Gilles

Station de Recherches Porcines 35590 L'Hermitage

\section{Intérêt et limites de la production de viandes de porc mâle entier}

Le gain de productivité résultant de l'abandon de la castration des porcs mâles n'est pas exploité en raison des odeurs sexuelles qui affectent les viandes de certains verrats. Des solutions partielles existent, qui pourraient dès à présent autoriser un premier développement de la production de mâles entiers. Les résultats récents de la recherche montent que l'on peut être optimiste quant aux possibilités futures d'avoir une maîtrise complète de l'apparition des odeurs sexuelles.

La production de viande de porc mâle entier présente un intérêt zootechnique bien établi. Outre la réduction de travail liée à l'absence de castration, les principaux avantages se situent au niveau du coût alimentaire et de la qualité des carcasses. La présence d'odeurs désagréables, qualifiées d'odeurs sexuelles, qui se manifestent lors de la cuisson des viandes de certains verrats, justifie que l'on continue le plus souvent à castrer systématiquement les jeunes mâles, se privant ainsi du potentiel élevé du porc mâle entier pour la production de viande.

Dans cet article nous exposerons les effets de la castration du porc mâle sur les performances zootechniques et la composition corporelle, ainsi que sur les caractéristiques qualitatives des viandes. Nous traiterons ensuite du problème posé par l'existence d'odeurs sexuelles dans les viandes de verrat, avant de présenter les différentes voies de recherche qui sont actuellement explorées pour tenter d'y remédier.

\section{Résumé}

La castration précoce du porc mâle entraîne des modifications importantes du métabolisme de l'animal, favorisant le dépôt des lipides au détriment de la fixation de protéines. La production de viandes de porc mâle entier permet ainsi une amélioration sensible de l'efficacité alimentaire et de la qualité des carcasses. Les viandes de porc mâle entier se caractérisent par des différences mineures de composition chimique des tissus, des graisses plus insaturées et par la présence des odeurs sexuelles. Le scatol et l'androsténone sont les deux déterminants majeurs de la manifestation de ces odeurs désagréables, mais les importances respectives de chacun de ces 2 composés ne sont pas encore clairement établies.

La transformation en produits de charcuterie offre de nombreuses possibilités d'utilisation des viandes défectueuses. Le tri sur la chaîne d'abattage des viandes présentant des odeurs sexuelles n'est possible que de façon grossière et imparfaite. Il n'existe pour l'instant aucun moyen de produire des verrats que l'on puisse garantir indemnes de défaut d'odeurs sexuelles. Les voies génétique et immunologique offrent des possibilités intéressantes qu'il faut explorer plus avant pour en démontrer la faisabilité sur les plans zootechnique et économique.

\section{1 / Performances de production}

La castration précoce des porcs mâles entraîne des modifications profondes de l'utilisation des nutriments énergétiques et azotés, ainsi que du métabolisme des protéines et des lipides. Il s'ensuit des différences importantes dans les performances de croissance et de composition corporelle entre animaux entiers et castrés.

\section{1 / Utilisation des nutriments énergétiques et azotés}

La part de l'énergie alimentaire perdue dans les fêces ou les urines n'est pas affectée par la castration du porc mâle (Holmes et al 1980). Selon Wiesemuller (1983), le besoin d'entretien du verrat serait supérieur de $10 \%$ à celui du castrat, mais ce résultat n'est pas confirmé par Noblet (1987). Pour une même quantité d'énergie métabolisable ingérée, l'énergie retenue est sensiblement identique mais se répartit différemment pour les 2 types sexuels, l'animal entier fixant moins d'énergie sous forme de lipides et davantage sous forme de protéines que son homologue castré (tableau 1). L'utilisation digestive de l'azote alimentaire n'étant pas modifiée par la castration, l'animal entier retient davantage d'azote que le castrat, avec un meilleur rendement (tableau 2). Pour les 2 types de nutriments, énergétique et azoté, les écarts entre les deux types sexuels sont très faibles en début de période de croissance $(25-30 \mathrm{~kg}$ ) alors qu'ils s'expriment pleinement en période de finition.

\section{2 / Métabolisme des protéines et des lipides}

Le turn-over des protéines musculaires est plus élevé chez le verrat que chez le castrat puisque, chez le premier, on observe à la fois une synthèse et une dégradation plus actives des protéines. Le bilan net reste toute- 
Tableau 1. Utilisation de l'énergie chez le porc mâle entier par rapport au castrat (\% de l'énergie métabolisable ingérée) (Noblet 1987, communication personnelle).

\begin{tabular}{|l|cc|cc|}
\hline \multicolumn{1}{|c|}{ Poids vif } & \multicolumn{2}{c|}{$\mathbf{2 5} \mathbf{~ k g}$} & \multicolumn{2}{c|}{$\mathbf{8 5} \mathbf{~ k g}$} \\
\hline \multicolumn{1}{|c|}{ Types sexuels } & Entier $\quad$ Castrat & Entier & Castrat \\
\hline Production de chaleur & 61 & 61 & 57 & 58 \\
Energie retenue : & & & & \\
- sous forme de protéines & 16 & 15 & 13 & 7 \\
- sous forme de lipides & 23 & 24 & 30 & 35 \\
\hline
\end{tabular}

Tableau 2. Utilisation de l'azote chez le porc mâle entier par rapport au castrat (\% de l'azote digestible) (Desmoulin et al 1974).

\begin{tabular}{|l|cc|cc|}
\hline \multicolumn{1}{|c|}{ Poids vif } & \multicolumn{2}{|c|}{$30 \mathrm{~kg}$} & \multicolumn{2}{c|}{$70 \mathrm{~kg}$} \\
\hline \multicolumn{1}{|c|}{ Types sexuels } & Entier & Castrat & Entier & Castrat \\
\hline \multirow{2}{*}{$\begin{array}{c}\text { Azote urinaire } \\
\text { Azote retenu }\end{array}$} & 41 & 41 & 49 & 59 \\
\hline
\end{tabular}

Le verrat produit à moindre coût une carcasse plus maigre. fois en faveur d'un dépôt plus important chez le verrat. Limplantation de testostérone ou de dihydrotestostérone aux castrats ne permet de restaurer que partiellement l'intensité du turn-over observée chez les mâles entiers (Mulvaney et al 1983a). Dans le tissu adipeux de verrat, la lipogenèse est moins active alors que la lipolyse est plus active que chez le castrat. L'implantation de testostérone chez le porc castré permet de retrouver les activités mesurées chez l'animal entier, alors que la dihydrotestotérone reste sans effet (Mulvaney et al 1983b), ce qui suggère un rôle important des oestrogènes dans le métabolisme du tissu adipeux. On sait en effet que, contrairement à la testostérone, la dihydrotestostérone n'est pas métabolisable en oestrogènes. Les expériences de supplémentation en stéroïdes de synthèse ont d'ailleurs montré que, dans l'espèce porcine, il est nécessaire d'associer androgènes et oestrogènes pour obtenir une amélioration significative des performances de croissance et de composition corporelle (Fowler et al 1978).

\section{3 / Performances zootechniques et composition corporelle}

De très nombreux travaux ont été consacrés à l'influence de la castration sur les performances zoo- techniques et la composition corporelle. Nous ne rapporterons ici que quelques exemples significatifs parmi l'ensemble des résultats disponibles dans la littérature (tableaux 3 et 4 ).

Lorsque les animaux sont alimentés à volonté, la vitesse de croissance du castrat est égale, voire supérieure, à celle du verrat. L'animal castré compense en effet sa moindre efficacité alimentaire par une plus grande consommation alimentaire. Par contre, dès lors que les quantités d'aliment allouées sont égales pour les 2 types d'animaux, la moindre efficacité alimentaire du castrat correspond à un ralentissement de la croissance par rapport à l'animal entier.

En raison de la présence d'un appareil génital pleinement développé, le rendement à l'abattage du verrat est plus faible de 1 à $3 \%$. Le tissu gras est moins développé alors que les tissus maigres sont plus importants chez l'animal entier que chez le castrat. On note en outre une répartition différente des masses musculaires, l'avant-train étant plus développé chez le verrat que chez le castrat (Walstra 1980).

Selon Desmoulin et Bonneau (1979), les avantages à attendre d'une production de porc mâle non castré peuvent se résumer à un gain de l'ordre de $30 \mathrm{~kg}$ d'aliment

Tableau 3. Performances zootechniques du porc mâle entier par rapport au castrat (castrat = indice 100).

\begin{tabular}{|l|l|c|l|}
\hline \multicolumn{1}{|c|}{ Critère } & Conditions d'alimentation & $\begin{array}{c}\text { Indice } \\
\text { du mâle entier }\end{array}$ & \multicolumn{1}{c|}{ Références } \\
\hline Consommation & ad libitum & 85 & Walstra 1980 \\
d'aliment & ad libitum & 88 & Fowler et al 1981 \\
\hline Gain moyen & ad libitum & 93 & Walstra 1980 \\
quotidien & semi ad libitum & 109 & Blair et English 1965 \\
& restreint & 103 & Walstra 1980 \\
\hline \multirow{2}{*}{ Indice } & ad libitum & 91 & Walstra 1980 \\
de consommation & semi ad libitum & 88 & Blair et English 1965 \\
& restreint & 93 & Walstra 1980 \\
\hline
\end{tabular}


Tableau 4. Composition corporelle du porc mâle entier par rapport au castrat (castrat = indice 100).

\begin{tabular}{|l|l|l|}
\hline \multicolumn{1}{|c|}{ Critère } & Indice du mâle entier & \multicolumn{1}{|c|}{ Références } \\
\hline Rendement à l'abattage & 99 & $\begin{array}{l}\text { Blair et English 1965 } \\
\text { Desmoulin 1973 } \\
\text { Walstra 1969 }\end{array}$ \\
\hline \multirow{2}{*}{ Epaisseur de lard } & 97 & Blair et English 1965 \\
& 98 & Desmoulin 1973 \\
& 81 & Walstra 1980 \\
\hline Pourcentage de tissus gras & 69 & Walstra 1969 \\
& 85 & Walstra 1980 \\
& 78 & Walstra 1980 \\
\hline \multirow{2}{*}{ Pourcentage de tissus maigres } & 85 & Walstra 1969 \\
& 82 & Walstra 1980 \\
& 111 & Walstra 1980 \\
\hline
\end{tabular}

Tableau 5. Qualité des viandes de verrat et de castrat : caractéristiques du tissu musculaire (BartonGade 1987).

\begin{tabular}{|l|c|c|c|}
\hline & Verrats & Castrats & Effet castration \\
\hline Composition chimique du semi-membranosus (\%) & & & \\
$\quad$ - Eau & 75.7 & 75.3 & $* * *$ \\
$\quad$ - Protéines & 21.5 & 21.8 & $*$ \\
$\quad$ - Lipides & 1.94 & 2.13 & $* * *$ \\
\hline Teneur en pigments du biceps femoris & 43.0 & 43.2 & NS \\
\hline Force de cisaillement du longissimus dorsi & 92.5 & 79.1 & $* * *$ \\
\hline
\end{tabular}

NS : Non significatif $\quad *: \mathrm{P}<0.05 \quad * * *: \mathrm{P}<0.001$

par porc produit et à une amélioration de 5 à 7 points du pourcentage de muscles de la carcasse. Compte tenu des profondes modifications de la structure génétique $\mathrm{du}$ troupeau porcin français intervenues au cours des dix dernières années, ces chiffres mériteraient d'être reprécisés.

\section{2 / Qualités des viandes}

\section{1 / Caractéristiques du tissu musculaire}

Le tissu musculaire des viandes de verrat est plus riche en eau et contient moins de protéines (tableau 5) et de matière sèche délipidée (Wood et Enser 1982). Le pourcentage de lipides intramusculaires est légèrement, mais significativement plus faible chez les verrats que chez les castrats (Desmoulin et al 1983 et tableau 5), cette différence n'étant observée, selon Wood et Enser (1982), que dans le cas d'un niveau d'alimentation bas.

Selon Barton-Gade (1987), la castration n'a que peu d'effet sur la fréquence des viandes exsudatives (PSE) ou sombres (DFD). Cependant, dans les cas où le temps d'attente avant abattage est long, la proportion de viandes DFD peut être plus élevée chez les verrats que chez les castrats, cet effet résultant d'une plus grande activité des mâles entiers (Moss et Robb 1978, Ellis et al 1983). Selon Barton-Gade (1987) la force de cisaillement des tissus musculaires est plus élevée chez les animaux entiers, ce qui est conforme aux observations de Martin et al (1968) et de Bonneau et al (1979) selon lesquelles les viandes de verrat sont moins tendres que celles de castrat.
Ainsi, les 2 types sexuels diffêrent pour les caractéristiques du tissu musculaire. Cependant, hormis le cas de la teneur en lipides, ces différences sont de faible amplitude et généralement considérées comme non significatives (Malmfors et Nilsson 1978, Desmoulin et al 1983).

\section{2 / Caractéristiques du tissu gras}

Le tissu gras des verrats est plus riche en eau et en protéines que celui des mâles castrés, alors qu'il contient moins de lipides (tableau 6). La teneur plus élevée en matière sèche délipidée dans le tissu gras dorsal est indicative d'une plus grande richesse en conjonctif (Wood et Enser 1982). Chez le verrat âgé, une partie du tissu gras dorsal de la région scapulaire s'est enrichie en conjonctif et considérablement appauvrie en lipides, jusqu'à présenter les caractéristiques du derme. Aux stades usuels d'abattage, les animaux à croissance normale ne sont cependant pas concernés par ce problème (Wood et al 1983 et $1985 \mathrm{~b}$ ).

Ainsi, les différences entre les caractéristiques des viandes de verrat et de castrat touchent essentiellement la composition chimique des tissus et le degré d'insaturation des graisses. Les différences de teneurs en eau, protéines et lipides sont assez faibles mais peuvent affecter le rendement de certaines fabrications comme le bacon (Ellis et al 1983). La plus grande insaturation des graisses résulte du fait que les verrats sont plus maigres ; il ne s'agit donc pas à proprement parler d'un caractère intrinsèque des viandes de mâle entier. 
Tableau 6. Qualité des viandes de verrat et de castrat : caractéristiques du tissu gras (Barton-Gade 1987).

\begin{tabular}{|l|r|r|c|}
\hline & Verrats & Castrats & Effet castration \\
\hline Composition chimique (\%) & & & \\
$\quad$ - Eau & 17.0 & 13.1 & $* * *$ \\
- Protéines & 4.7 & 3.6 & $* * *$ \\
- Lipides & 78.1 & 83.4 & $* * *$ \\
\hline Composition en acides gras (\%) & & & \\
C16 & 24.9 & 26.1 & $* *$ \\
C18 & 13.5 & 13.6 & NS \\
C18:1 & 41.8 & 43.3 & $* * *$ \\
C18:2 & 11.8 & 9.3 & $* * *$ \\
\% acides gras insaturés & 58.6 & 57.3 & $* * *$ \\
Indice d'iode & 66.6 & 63.3 & $* * *$ \\
\hline Teneur en scatol (ppm) & 0.07 & 0.05 & $*$ \\
\hline
\end{tabular}

NS : Non significatif $\quad * * *: P<0.001$

L'ensemble des caractéristiques évoquées ci-dessus constituent des défauts qualitatifs relativement mineurs en comparaison des problèmes posés par la présence des odeurs sexuelles.

\section{3 / Les odeurs sexuelles des viandes de verrat}

Les viandes

de certains verrats dégagent à la cuisson des odeurs désagréables : elles sont impropres à la consommation en frais.

Des odeurs désagréables, qualifiées d'odeurs sexuelles, se manifestent fréquemment lors de la cuisson des viandes de verrats âgés. Aux stades usuels d'abattage, les mâles entiers ne présentent pas tous ces odeurs, la proportion d'animaux concernés variant de 10 à $75 \%$ selon les auteurs (Williams et al 1963, Desmoulin et al 1971, Rhodes et Patterson 1971, Malmfors et Hansson 1974). Compte tenu d'une telle variabilité dans la fréquence des odeurs sexuelles, et aussi de la diversité des habitudes culinaires selon les pays, les enquêtes d'acceptabilité des viandes de jeunes verrats auprès du grand public donnent des résultats parfois divergents (Malmfors et Lundstrom 1983), certains allant jusqu'à conclure à l'absence de différence entre types sexuels. Selon Desmoulin et al (1982), les critiques portées par des consommateurs français sur l'odeur de cuisson des viandes concernent $38 \%$ des verrats contre $6 \%$ des témoins, mâles castrés et femelles ; pour le goût des viandes, les écarts sont moins prononcés : $23 \%$ de critiques pour les mâles entiers contre $12 \%$ pour les témoins.

Après avoir fait le point des connaissances sur les composés responsables des odeurs sexuelles, nous évoquerons les principales voies de recherches actuellement explorées pour tenter de remédier à ce problème.

\section{3 / Situation du problème posé par les odeurs sexuelles des viandes de porc mâle entier}

\section{1 / Les composés responsables des odeurs sexuelles}

Deux composés, l'androsténone et le scatol, localisés dans la fraction insaponifiable des graisses, sont considérés comme les principaux déterminants des odeurs sexuelles des jeunes verrats.
L'androsténone est un stéroïde d'origine testiculaire à odeur urinaire prononcée, qui se stocke dans les graisses à des concentrations à l'ordre du ppm (partie par million). Les coefficients de corrélation entre la concentration en androsténone du tissu adipeux et l'intensité des odeurs sexuelles sont compris entre 0,4 et 0,7 (tableau 7). Compte tenu de l'imprécision inhérente à l'appréciation subjective des odeurs, une telle liaison indique que l'androsténone est un déterminant essentiel des odeurs sexuelles.

Le scatol est un produit de la dégradation du tryptophane par la flore intestinale. La mise en évidence de son rôle dans la manifestation des odeurs sexuelles est difficile car les méthodes de dosage de ce composé dans les graisses sont peu précises (Hansson et al 1980) ou peu spécifiques (Mortensen et Sorensen 1984), alors que les concentrations observées sont assez faibles (tableau 6). Il semble bien cependant que ce composé soit un déterminant important des odeurs sexuelles (Lundstrom et al 1987).

\section{2 / Facteurs de variation des concentrations en scatol des graisses du verrat}

Compte tenu du caractère récent de la mise en évidence du rôle du scatol, et des problèmes méthodologiques évoqués plus haut (manque de spécificité du dosage) on sait peu de choses sur la régulation de la synthèse et du stockage de ce composé chez le porc. On constate que les verrats présentent des teneurs en scatol du tissu gras significativement supérieures à celles observées chez les castrats et les femelles (Hansson et al 1980, Lundstrom et al 1987, Barton-Gade 1987). Comme l'indiquent Lundstrom et al (1987), le stockage de scatol dans les graisses est vraisemblablement dépendant de la production des stérö̈des testiculaires, sans que l'on puisse fournir à l'heure actuelle d'explication satisfaisante à ce phénomène.

Les teneurs en scatol des graisses de verrat differrent significativement entre élevages (Pedersen et al 1986) ou entre loges d'un même bâtiment (Hansson et al 1980), ce qui indique une importante influence de l'environnement et/ou de l'état sanitaire des animaux. La production de scatol pourrait être favorisée dans le cas de régimes riches en fibres qui stimulent les fermentations intestinales (Lundstrom et al 1987). La supplémenta- 
tion de l'aliment en tryptophane n'entraine aucune élévation des teneurs en scatol (Pedersen et al 1986) ou de la fréquence des odeurs sexuelles (Bonneau et Desmoulin 1981), car le tryptophane de synthèse, entièrement disponible, est complètement absorbé au niveau de l'intestin grêle. On peut penser qu'il n'en irait pas de même dans le cas de régimes riches en tryptophane à disponibilité plus réduite (ex. farines de luzerne), mais cela reste à démontrer.

\section{3 / Facteurs de variation des concentrations en androsténone des graisses du verrat}

Un exposé détaillé des voies de biosynthèse et du métabolisme de l'androsténone et des facteurs de variation de la teneur en androsténone des graisses peut être trouvé dans les revues bibliographiques les plus récentes (Bonneau 1982, Brooks et Pearson 1986). Nous ne ferons que rappeler brièvement les points les plus importants.

L'intensité de la synthèse d'androsténone est d'abord faible chez le jeune animal, puis augmente brutalement au moment de l'établissement de la puberté, en même temps que s'élève la production des autres stéroïdes d'ori-

Figure 1. Un exemple de distribution des teneurs en androsténone du tissu adipeux (Bonneau et Desmoulin 1979).

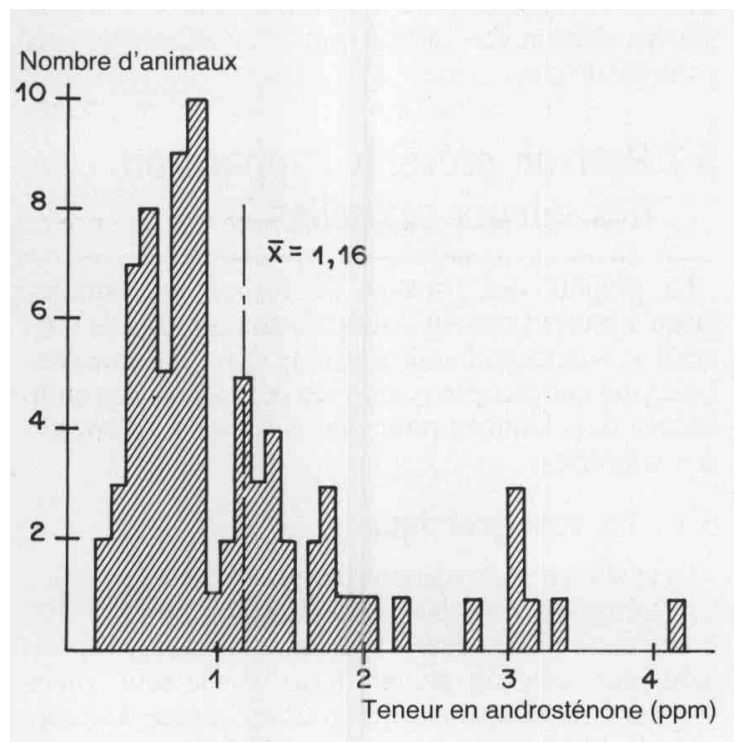

Teneur en adrosténone

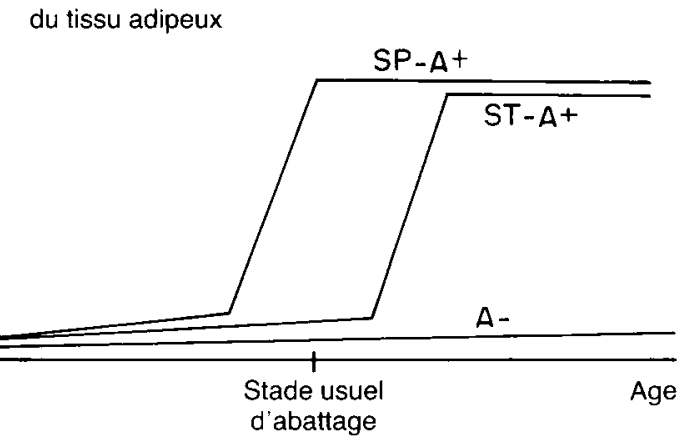

gine testiculaire (androgènes et oestrogènes) qui sont responsables des bonnes performances zootechniques de l'animal entier. Ainsi, le testicule de verrat produit à la fois des composés "utiles" (androgènes et oestrogènes) et un composé "gênant" (androsténone). Or ce sont les mêmes systèmes de régulation qui gouvernent l'intensité de la synthèse de ces deux familles de stéroïdes. ll est donc difficile de se débarrasser des uns tout en préservant les autres.

Aux stades usuels d'abattage, la teneur en androsténone des graisses est très variable selon les animaux (figure 1). Pour comprendre l'origine de ce phénomène il faut considérer les profils individuels d'évolution avec l'âge (ou le poids) de la teneur en androsténone du tissu adipeux (figure 2). Les différences de précocité sexuelle entre animaux fournissent une première explication à la variabilité des teneurs : les animaux sexuellement précoces (SP) ont pu exprimer pleinement leur potentiel de production d'androsténone avant l'abattage alors que les porcs sexuellement tardifs (ST) présentent des teneurs en androsténone encore faibles. Une deuxième explication importante tient aux différences entre individus pour le potentiel de production d'androsténone. Il existe en effet des individus $\left(\mathrm{A}^{-}\right.$sur la figure 2 ) qui présentent des teneurs en androsténone constamment faibles, même après la puberté. Chez ces animaux, l'élévation péripubertaire de la teneur en androsténone a bien lieu mais reste limitée à des niveaux de concentration faibles, inférieurs à 0,5 ppm (Bonneau et al 1987).

Dans l'état actuel des connaissances, on ne sait pas lequel des 2 composés, androsténone ou scatol, est le déterminant essentiel des odeurs sexuelles. Les études ayant tenté de faire la part des rôles respectifs de l'androsténone et du scatol (Walstra et al 1986, Lunds-
Figure 2. Trois types d'évolution avec l'âge de la teneur en androsténone du tissu adipeux (Bonneau 1984).

SP : animaux sexuellement précoces

ST : animaux sexuellement tardifs

A : teneur en androsténone élevée $\left(A^{+}\right)$ou

constamment faible $\left(A^{-}\right)$

Tableau 7. Coefficient de corrélation entre la teneur en androsténone des graisses et l'intensité des odeurs sexuelles.

\begin{tabular}{|l|c|c|}
\hline \multicolumn{1}{|c|}{ Références } & Nombre de données & Coefficient de corrélation \\
\hline Patterson et Stinson 1971 & 23 & 0.76 \\
Maarse et al 1972 & 17 & 0.69 \\
Newell et al 1973 & 44 & 0.53 \\
Malmfors et Andresen 1975 & 111 & 0.51 \\
Thompson et Pearson 1977 & 17 & 0.46 \\
Malmfors et al 1978 & 104 & 0.43 à 0.66 \\
Bonneau et al 1979 & 36 & 0.41 à 0.57 \\
Bonneau et Desmoulin 1979 & 77 & 0.44 \\
Jonsson et Andresen 1979 & 648 & 0.50 \\
Otto et Behm 1979 & 75 & 0.65 \\
Forland et al 1980 & 44 & 0.47 \\
Hansson et al 1980 & 84 & 0.60 \\
Otto et Behm 1981 & 416 & 0.65 \\
\hline
\end{tabular}


trom et al 1987) souffrent de faiblesses méthodologiques importantes. L'hypothèse la plus vraisemblable est que, selon le type de production considéré, l'un ou l'autre des composés a un rôle prédominant. Dans le cas d'animaux abattus jeunes, avant leur maturité sexuelle, les teneurs en androsténone sont faibles et on peut raisonnablement penser que le scatol jouerait alors un rôle déterminant. Par contre, lorsque les animaux sont abattus à des stades plus tardifs, l'androsténone pourrait devenir le responsable majeur des odeurs sexuelles. Les teneurs moyennes en androsténone du tissu adipeux, et donc l'importance de la contribution de ce composé à la manifestation des odeurs sexuelles, varient aussi beaucoup en fonction du type génétique des animaux. Seule une étude comparative à grande échelle, intégrant la diversité des systèmes de production et des habitudes alimentaires des différents pays européens, permettra d'apporter une réponse concluante à cette question.

Plusieurs approches sont possibles pour tenter d'apporter une solution au problème posé par les odeurs sexuelles des viandes de verrat. On peut d'abord explorer les possibilités d'emploi des viandes défectueuses, ou encore s'efforcer de prévenir l'apparition des odeurs sexuelles chez l'animal pendant la phase d'engraissement. Ces deux voies de recherches seront évoquées successivement.

\section{4 / Peut-on utiliser les viandes présentant des odeurs sexuelles?}

\section{1 / Transformation en produits de charcuterie}

Un certain nombre de travaux ont établi l'influence bénéfique de la transformation en produits de charcuterie sur l'acceptabilité des viandes porteuses d'odeurs sexuelles (Williams et al 1963, Pearson et al 1971, Walstra 1974, Bonneau et al 1979). Une partie de l'androsténone stockée dans le tissu gras disparaît au cours de la transformation, dans une proportion qui dépend du processus technologique employé (Bonneau et al 1980). Par ailleurs le seuil de teneur en androsténone au-delà duquel le produit est considéré comme inacceptable est plus élevé (Desmoulin et al 1982). A ces deux influences favorables s'ajoute, dans le cas des produits de mélange (saucisses, saucissons, pâtés, etc...) la possibilité de diluer les viandes défectueuses par des viandes indemnes. Il apparaît ainsi que la transformation en produits de charcuterie permet d'utiliser les viandes présentant des odeurs sexuelles, dans des conditions qu'il faudrait encore préciser par une étude plus systématique de l'influence des divers processus technologiques sur le devenir de l'androsténone stockée et l'acceptabilité des produits.
La sélection contre les odeurs sexuelles est efficace mais ne doit pas se faire au détriment des performances de croissance.

méthodes "olfactives" consistant à chauffer du tissu gras pour essayer de déceler l'odeur de verrat ne sont pas utilisables dans les conditions pratiques de l'abattoir (Bonneau et Desmoulin 1975). Les méthodes de mesure de l'androsténone dans le tissu adipeux sont longues et onéreuses. Après plus de 10 ans d'efforts visant à automatiser le dosage de l'androsténone dans les graisses, les chercheurs hollandais ont arrêté leurs travaux. Le dosage automatisé du scatol, mis au point par Mortensen et Sorensen (1984), permettrait, selon ces auteurs, de suivre les cadences usuelles d'abattage ; il reste cependant à démontrer que cette méthode est suffisamment fiable pour assurer une protection efficace des consommateurs (cf. paragraphe 3.1).

L'observation du développement des glandes annexes de l'appareil génital permet de différencier les animaux impubères (qui sont à coup sûr indemnes d'odeurs liées à l'androsténone et probablement aussi de celles liées au scatol) des animaux pubères (Bonneau et Russeil 1984). Ces derniers présentent à des degrés variables des odeurs sexuelles mais une proportion non négligeable d'entre eux est, en fait, également indemne. En pratique, la seule mesure de la longueur des glandes bulbouréthrales (encore appelées glandes de Cowper), permet de trier une population de porcs d'abattage en 2 sous-populations d'effectif sensiblement égal. La moitié environ des animaux, qui ont les plus petites glandes, peuvent être classés "indemnes" et utilisés pour la consommation en frais. Les $50 \%$ restant sont douteux et, en l'absence d'autre méthode de détection plus précise, doivent être dirigés vers la transformation en produits de charcuterie.

\section{5 / Peut-on prévenir l'apparition des odeurs sexuelles?}

La plupart des travaux de recherche conduits jusqu'à présent ont été consacrés aux moyens de prévenir le stockage d'androsténone dans les graisses. En ce qui concerne le scatol, les connaissances sont encore trop limitées pour que l'on puisse proposer des solutions.

\section{1 / La voie génétique}

La teneur en androsténone des graisses est un caractère fortement héritable (Jonsson et Andresen 1979, Bonneau et Sellier 1986, Willeke et al 1987). Cependant, une sélection aveugle basée sur le seul critère "teneur en androsténone des graisses" risque d'entraîner une diminution de la production des hormones stéroïdes androgènes et oestrogènes et, en conséquence, une détérioration des performances et une maturation sexuelle plus tardive. Willeke et al (1987) ont effectivement observé un retard à la puberté chez les femelles appartenant à une "lignée basse" pour la teneur en androsténone du tissu gras.

Il existe des animaux qui présentent à la fois de faibles potentialités de production d'androsténone et une synthèse normale des hormones "utiles" androgènes et œstrogènes (Bonneau et al 1987). Dans une expérience préliminaire, il a été établi que la sélection de tels animaux est possible, permettant de réduire très efficacement les niveaux de teneur en androsténone des graisses tout en sauvegardant le développement sexuel du verrat (tableau 8). Il reste à vérifier si une telle sélection permettrait de préserver les performances de croissance et de composition corporelle et qu'elle n'aurait pas, par ailleurs, de conséquence défavorable sur les performances de reproduction des femelles appararentées. 


\section{2 / La voie immunologique}

Plusieurs équipes de recherche se sont efforcées de "vacciner" des verrats contre l'androsténone (Daniel et al 1984, Williamson et al 1985) ou contre des précurseurs immédiats de ce stéroïde (Brooks et al 1986). Cette procédure est a priori très séduisante car elle permettrait d'éliminer l'androsténone tout en préservant les sécrétions des hormones "utiles", androgènes et oestrogènes. Les résultats obtenus ont été très variables, certains constatant une réduction significative des teneurs en androsténone alors que d'autres n'observent aucune diminution. Même dans les cas les plus favorables, la réduction des teneurs n'est pas suffisante pour garantir une protection suffisante du consommateur visà-vis des odeurs sexuelles. Les recherches continuent pour essayer de trouver des méthodes de vaccination plus efficaces permettant d'obtenir un résultat plus complet.

La castration tardive des verrats, en fin de période d'engraissement, permettrait de profiter de l'effet bénéfique des hormones androgènes et oestrogènes pendant la plus grande partie de la vie productive de l'animal tout en évitant les odeurs sexuelles. Des expériences utilisant la castration chirurgicale des verrats ont montré qu'il suffit d'intervenir deux à trois semaines avant la date prévue pour l'abattage (Bonneau et al 1982), mais cette méthode n'est pas utilisable dans la pratique de l'élevage. L'auto-immunisation des animaux contre la LHRH (une hormone de l'hypothalamus qui contrôle la secrétion de l'hormone hypophysaire LH, qui elle-même régule la synthèse des stéroïdes testiculaires) permet de réduire les concentrations en androsténone des graisses à des niveaux très faibles, semblables à ceux mesurés chez les mâles castrés précocément ou chez les femelles (Caraty et Bonneau 1986, figure 3). Cependant l'adjuvant d'immunisation mis en œuvre dans cette expérience n'est pas utilisable dans la pratique de l'élevage industriel. Il faut donc mettre au point une méthode de vaccination anti-LHRH plus simple. Une fois cet objectif atteint, il restera à évaluer les conséquences de l' "immuno-castration" ainsi réalisée sur les performances zootechniques des animaux.

En résumé, la sélection et l'immunocastration sont des méthodes séduisantes. Leur efficacité étant maintenant
Figure 3. Evolution des teneurs en androsténone du tissu adipeux après immunisation active contre le LH-RH (Caraty et Bonneau 1986).

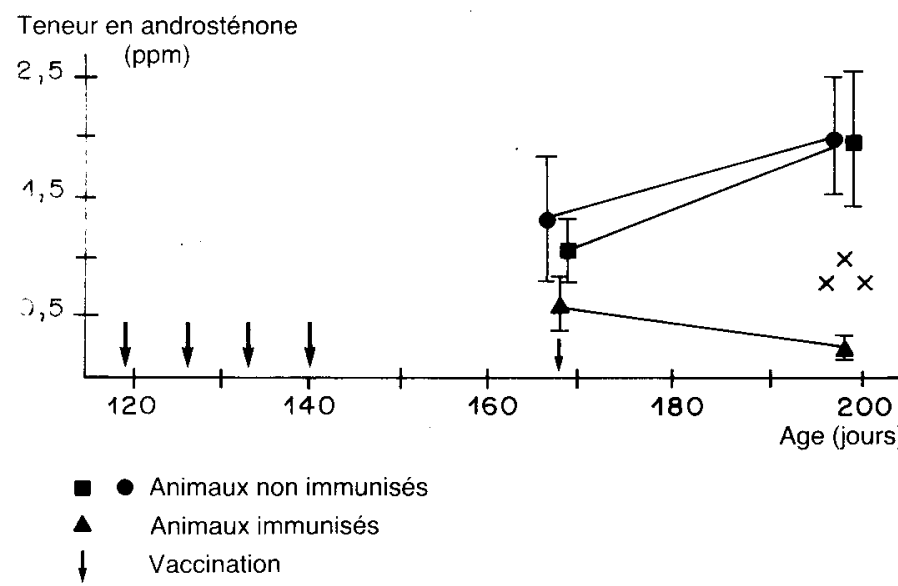

démontrée, il reste à faire la preuve de leur faisabilité "zootechnique" et "économique".

\section{Conclusion}

Lélevage des porcs mâles non castrés pour la production de viande permettrait un gain de productivité important, mais cette possibilité n'est pas exploitée à l'heure actuelle, car une partie des viandes de verrat présentent des odeurs sexuelles. Le scatol et l'androsténone sont les deux déterminants majeurs de la manifestation de ces odeurs désagréables, mais les importances respectives de chacun de ces composés ne sont pas encore clairement établies. Les facteurs de variation de la teneur en androsténone des graisses sont maintenant bien connus, les facteurs génétiques apparaissant déterminants.

La transformation en produits de charcuterie offre de nombreuses possibilités d'utilisation des viandes défec-

Tableau 8. Réponses des verrats à une sélection combinée pour une faible teneur en androsténone des graisses et un faible ou fort développement testiculaire (Lignée témoin -NS- = indice 100) (Sellier et al 1987).

\begin{tabular}{|c|c|c|c|c|}
\hline \multirow[b]{2}{*}{$\begin{array}{l}\text { Caractères mesurés } \\
\text { sur les descendants }\end{array}$} & \multicolumn{4}{|c|}{ Critères de sélection des pères } \\
\hline & $\begin{array}{l}\text { Teneur en } \\
\text { androsténone } \\
\text { Développement } \\
\text { testiculaire }\end{array}$ & $\begin{array}{l}\text { Faible } \\
\text { Faible }\end{array}$ & $\begin{array}{l}\text { NS* }^{*} \\
\text { NS* }^{*}\end{array}$ & $\begin{array}{l}\text { Forte } \\
\text { Fort }\end{array}$ \\
\hline \multicolumn{2}{|c|}{ 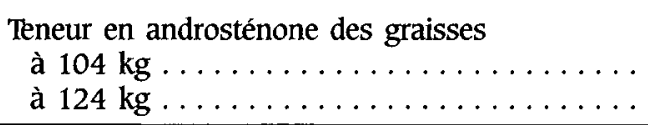 } & $\begin{array}{l}70^{b} \\
60^{b}\end{array}$ & $\begin{array}{l}100^{\mathrm{a}} \\
100^{\mathrm{a}}\end{array}$ & $\begin{array}{l}54^{b} \\
65^{b}\end{array}$ \\
\hline \multicolumn{2}{|c|}{$\begin{array}{l}\text { Développement de l'appareil génital } \\
\begin{aligned} \text { à } 104 \mathrm{~kg} \text { - développement testiculaire } \ldots \ldots \\
\text { à } 124 \mathrm{~kg} \text { - poids des testicules } \ldots \ldots \ldots \\
\text { - poids des glandes de Cowper . . . . } \\
\text { - longueur des glandes de Cowper }\end{aligned}\end{array}$} & $\begin{array}{l}93^{b} \\
86^{b} \\
95^{a} \\
95^{b}\end{array}$ & $\begin{array}{l}100^{\mathrm{a}} \\
100^{\mathrm{a}} \\
100^{\mathrm{a}} \\
100^{\mathrm{a}}\end{array}$ & $\begin{array}{l}97^{\mathrm{a}} \\
100^{\mathrm{a}} \\
101^{\mathrm{a}} \\
102^{\mathrm{a}}\end{array}$ \\
\hline
\end{tabular}


tueuses. Le tri des viandes présentant des odeurs sexuelles n'est possible que de façon grossière et imparfaite. Cependant, même si l'on considère l'hypothèse maximaliste où aucun porc mâle ne serait castré, ce n'est qu'un quart de la production (la moitié des mâles) qu'il faudrait écarter des circuits de distribution en frais. Dans les situations où il est supportable de diriger une partie des longes vers la transformation, le tri des carcasses mâles en fonction de la longueur des glandes de cowper pourrait permettre de développer davantage qu'à l'heure actuelle l'élevage de porcs mâles non castrés.

Il n'existe pour l'instant aucun moyen de produire des verrats que l'on puisse garantir indemnes d'odeurs sexuelles. Les voies génétique et immunologique offrent des possibilités intéressantes qu'il faut explorer plus avant pour en démontrer la faisabilité sur les plans zootechnique et économique.

\section{Principales références bibliographiques}

(La liste complète des références citées est disponible auprès de l'auteur)

BARTON-GADE P.A., 1987. Meat and fat quality in boars, castrates and gilts. Livest. Prod. Sci., 16, 187-196.

BONNEAU M., 1982. Compounds responsible for boar taint with special emphasis on androstenone : a review. Livest. Prod. Sci., 9, 687-705.

BONNEAU M., RUSSEIL P., 1984. Estimation à l'abattoir des odeurs sexuelles des viandes de porcs mâles entiers par examen de l'appareil génital. Journées Rech. Porcine en France, 16, 81-90.
BROOKS R.I., PEARSON A.M., 1986. Steroid hormone pathways in the pig, with special emphasis on boar odor : a review. J. Anim. Sci., 62, 632-645.

CARATY A., BONNEAU M., 1986. Immunisation active du porc mâle contre la gonadolibérine : effets sur la sécrétion d'hormones gonadotropes et sur la teneur en 5 -androst-16-ène-3-one du tissu adipeux. C. R. Acad. Sc. Paris, Série III, 303, 673-676.

DESMOULIN B., BONNEAU M., 1979. Aptitudes à l'emploi des viandes de porcs mâles entiers. Techni Porc, 2, 15-54.

DESMOULIN B., BONNEAU M., FROUIN A., BIDARD J.P., 1982. Consumer testing of pork and processed meat from boars : the influence of fat androstenone level. Livest. Prod. Sci., 9, 707-715.

DESMOULIN B., GIRARD JP. BONNEAU M., FROUIN A. 1983. Aptitudes à l'emploi des viandes porcines selon le type sexuel, le système d'alimentation et le poids d'abattage. Journées Rech. Porcine en France, 8, 89-98.

LUNDSTROM K., MALMFORS B., MALMFORS G., STERN S. PETERSSON H., MORTENSEN A.B., SORENSEN S.E., 1987. Skatole, androstenone and taint in boars fed two different diets. Livest. Prod. Sci., 18, 55-67.

MALMFORS B., LUNDSTROM K., 1983. Consumer reactions to boar meat - a review. Livest. Prod. Sci., 10, 187-196.

SELLIER P., BONNEAU M., GRUAND ]., 1987. Relations génétiques entre la teneur en androsténone du gras chez le verrat et le développement de l'appareil génital mâle et femelle. Journées Rech. Porcine en France, 19, 33-40

WALSTRA P., 1974. Fattening of young boars : quantification of positive and negative aspects. Livest. Prod. Sci., 1, 187-196.

WILLEKE H., CLAUS R., MULLER E., PIRCHNER F, KARG H., 1987. Selection for high and low level of 5 - androst-16-en-3-one in boars. I. Direct and correlated response of endocrinological traits. J. Anim. Breed. Genet., 104, 64-73.

WILLIAMSON E.D., PATTERSON R.L.S., BUXTON E.R., MITCHELL K.G., PARTRIDGE I.G., WALKER N., 1985. Immunization against 5 - androstenone in boars. Livest. Prod. Sci., 12, 251-264. 